\title{
A Particle Swarm Optimization Based SAR Motion Compensation Algorithm for Target Image Reconstruction
}

\author{
Salih Uḡur \\ Meteksan Savunma A.S. \\ Ankara, Turkey \\ Bilkent University \\ Electrical and Electronics Engineering Department \\ Ankara, Turkey
}

\author{
Orhan Arıkan \\ Bilkent University \\ Electrical and Electronics Engineering Department \\ Ankara, Turkey
}

\begin{abstract}
A new SAR motion compensation algorithm is proposed for robust reconstruction of target images even under large deviations of the platform from intended flight path. Phase error due to flight path deviations is estimated as a solution to an optimization problem in terms of the positions of the reflectivity centers of the target. Particle swarm optimization is used to obtain phase error estimates efficiently. The quality of the reconstructions is demonstrated by using simulation studies.
\end{abstract}

\section{INTRODUCTION}

Phase errors caused by uncompensated platform movements cause significant distortion on SAR images. There are several methods that are known as autofocus techniques proposed to correct SAR phase errors and provide reconstructions with reduced distortion. One of the most commonly used technique is the Phase Gradient Autofocus (PGA) [1], [2]. To further improve the reconstruction quality that can be achieved for targets with closely spaced reflectivity centers, we propose a new method to autofocus the SAR images. Since such targets are common among the targets of interest, the proposed technique addresses important SAR applications. The proposed autofocus technique models target of interest with few scattering centers with unknown positions. Then, cast the motion related phase errors as an estimation problem. Here we propose to use a Particle Swarm Optimization (PSO) [3] based estimation technique to obtain a globally optimal phase error estimate. A similar approach to our modeling of targets as multiple point scatterers and applying PSO to find their parameters, is also used in an RF tomography problem to estimate sensor positions using PSO [4]. Previously, novel methods to improve SAR autofocusing using PSO algorithm have been also suggested [5], [6]. In those methods, the phase error is modeled and its model parameters are estimated. In contrast to those previous techniques, we do not model the phase error, but model the targets as multiple point scatterers and then choose the target model parameters that minimize the error between the amplitudes of returned signal and the signal corresponding to point scatterers. Finally, the phase error is obtained from the phase differences of these two signals.

The formulation of the received SAR signal under motion errors will be given in section two. Then the details of the method of phase estimation using the PSO technique will be presented. In section four, the performance of the proposed autofocusing technique will be investigated by a simulation study. At the end, section five contains conclusion.

\section{THE EFFECT OF MOTION ERRORS ON SAR DATA}

In SAR processing, raw SAR data is pulse compressed both in range and cross-range directions. Pulse compression in range direction conveys uncompensated motion errors directly as range position errors. However, the magnitude of uncompensated platform motion errors (which causes phase errors in raw SAR signal) is generally much less than the range resolution of SAR sensors. Therefore the assumption that uncompensated platform motion errors in raw SAR signal do not distort the image in the range direction, is a reasonable one. But small phase errors degrade the phase information along the cross-range direction which is used for cross-range compression. Hence, the motion related errors typically cause blurred images in the cross-range direction. In order to alleviate phase error effects on SAR images, here we focus on the SAR signal along the cross-range direction. The demodulated and range compressed stripmap SAR signal for a point target is given by the formula [7]:

$$
s(t, \eta)=A_{0} p_{r}\left(t-\frac{2 R(\eta)}{c}\right) w_{a}\left(\eta-\eta_{c}\right) e^{-j \frac{4 \pi}{\lambda} R(\eta)} .
$$

Here, $t$ is fast time, $\eta$ is slow time, $\eta_{c}$ is beam center crossing time, $\lambda$ is wavelength, $A_{0}$ is complex amplitude of the point scatterer, $p_{r}(t)$ is compressed signal profile in range direction, $R(\eta)$ is slant range at time $\eta$ and $w_{a}(\eta)$ is azimuth envelope caused by antenna azimuth pattern. We assume zero squint angle for clarity, so the beam center crossing time $\left(\eta_{c}\right)$ coincides with the point target position in azimuth time. For $N$ point reflectors that are located at the same range bin, but 
at different azimuth locations, the signal takes the form:

$$
s(t, \eta)=\sum_{i=1}^{N} A_{i} p_{r}\left(t-\frac{2 R_{i}(\eta)}{c}\right) w_{a}\left(\eta-\eta_{i}\right) e^{-j \frac{4 \pi}{\lambda} R_{i}(\eta)},
$$

where the point reflectors are indexed by $i$. Because phase errors affect the SAR image mainly in the azimuth direction, we take cross section of SAR signal along azimuth axis at the range bin where the point reflectors are located. Here we neglect effects of other reflectors that are residing in neighboring range bins. This is acceptable when sidelobes caused by range compression is reduced to an appropriately low levels by using suitable windowing functions. Also neglecting range migration effects, we take $p_{r}(t)$ to be constant. Moreover, we neglect the antenna aperture effects and take $w_{a}(\eta)$ equal to one for targets that are located in the beam coverage of the antenna. These assumptions lead to simpler formulation and easier understanding of the underlying phenomena and do not cause significant inaccuracies. They can also be taken into account at a later stage of the formulation. Under these assumptions, the SAR signal cross section in a range bin (where point targets reside) along the azimuth direction becomes:

$$
s(\eta)=\sum_{i=1}^{N} A_{i} e^{-j \frac{4 \pi}{\lambda} R_{i}(\eta)} .
$$

The slant range of the $i^{t h}$ point reflector at azimuth time $\eta$ is:

$$
R_{i}(\eta)=R_{t i}(\eta)+\Delta R_{e i}(\eta),
$$

where, $R_{t i}(\eta)$ is the actual slant range between the $i^{\text {th }}$ point reflector and the platform at azimuth time $\eta$, and $\Delta R_{e i}(\eta)$ is the slant range error term caused by the uncompensated motion errors. For large $R_{o}$ (range of closest approach) compared to swath widths, the actual slant range can be very closely approximated as:

$$
R_{t i}(\eta) \simeq R_{o}+\frac{1}{2 R_{o}}\left(\eta-\eta_{i o}\right)^{2} v^{2} .
$$

Because all point reflectors are located in the same range bin, $R_{o}$ is the same for all targets and so it does not have the $i$ subscript. In the above formula $\eta_{i o}$ is the slow time when the slant range between the platform and the $i^{t h}$ point target equals $R_{o}$ and $v$ is the platform speed. Defining the reference signal $s_{r e f}(\eta)$ as:

$$
s_{r e f}(\eta)=e^{j \frac{2 \pi v^{2}}{\lambda R_{o}} \eta^{2}}
$$

and multiplying the original signal with the reference signal, we get:

$$
s_{r}(\eta)=\sum_{i=1}^{N} C_{i} e^{j \frac{4 \pi v^{2} \eta_{i o}}{\lambda R_{o}} \eta} e^{j \phi_{e i}(\eta)}
$$

where,

$$
C_{i}=A_{i} e^{-j \frac{4 \pi}{\lambda} R_{o}} e^{-j \frac{2 \pi v^{2}}{\lambda R_{o}} \eta_{i o}^{2}} .
$$

Here $\phi_{e i}(\eta)$ (equals to $-(4 \pi / \lambda) \Delta R_{e i}(\eta)$ ) is the phase error contribution due to the $i^{t h}$ point reflector. For SAR sensors with large $R_{o}$ 's compared to swath widths, differences between phase errors of different targets can be neglected and therefore we can drop the $i$ subscript from the phase errors $\phi_{e i}(\eta)$. Because the main aim of this study is to correct the distorted images caused by phase errors of closely spaced reflectors, neglecting the differences between phase errors of closely spaced reflectors do not cause significant errors. Under the assumption of the same phase error for all reflectors at the same range bin, the signal becomes:

$$
s_{r}(\eta)=\sum_{i=1}^{N} C_{i} e^{j \frac{4 \pi v^{2} \eta_{i o}}{\lambda R_{o}} \eta} e^{j \phi_{e}(\eta)} .
$$

\section{Phase ERror Estimation}

The demodulated and range compressed signal affected by the motion related phase error and multiplied by $s_{\text {ref }}(\eta)$ can be re-written in a more compact form as:

$$
s_{r}(\eta)=\left(\sum_{i=1}^{N} C_{i} e^{j \omega_{i} \eta}\right) e^{j \phi_{e}(\eta)},
$$

where, $\omega_{i}=\left(4 \pi v^{2} \eta_{i o} / \lambda R_{o}\right)$. Note that, the contribution of a point reflector with complex amplitude $A_{i}$ and located at $\eta_{i o}$ is a complex exponential with complex amplitude $C_{i}$ and frequency $\omega_{i}$, which is proportional to the position of the point reflector in the azimuth direction. This result is also compatible with the result that the multiplication with the reference signal $s_{r e f}(\eta)$ provides the cancelation of $\eta^{2}$ terms in the exponent, and thus, provides a Fourier Transform relation between the resultant signal and the azimuth positions of point targets. Here, note that the magnitude of the signal, $s_{r}(\eta)$, is the magnitude of the sum of $N$ complex exponentials with complex amplitudes, $C_{i}=\left|C_{i}\right| e^{j L C_{i}}$,

$$
\left|s_{r}(\eta)\right|=\left|\sum_{i=1}^{N}\right| C_{i}\left|e^{j \omega_{i} \eta+\angle C_{i}}\right|
$$

and phase of the signal $s_{r}(\eta)$ is,

$$
\angle s_{r}(\eta)=\angle\left(\sum_{i=1}^{N}\left|C_{i}\right| e^{j \omega_{i} \eta+\angle C_{i}}\right)+\phi_{e}(\eta) .
$$

The phase error term does not affect the magnitude of the signal $s_{r}(\eta)$, but only affects the phase of it. We can try to estimate the phase error by finding a set of $N$ complex exponentials whose sum has a magnitude that is equal to the magnitude of the $s_{r}(\eta)$ :

$$
\begin{gathered}
\tilde{s}_{r}(\eta)=\sum_{k=1}^{N}\left|D_{k}\right| e^{j \omega_{k} \eta+\angle D_{k}}, \\
\left|\tilde{s_{r}}(\eta)\right|=\left|s_{r}(\eta)\right|,
\end{gathered}
$$

where $D_{k}=\left|D_{k}\right| e^{j \angle D_{k}}$ is the complex amplitude of the $k^{t h}$ complex exponential $e^{j \omega_{k} \eta}$. Then corresponding phase error estimate is the difference between the phases of $s_{r}(\eta)$ and $\tilde{s_{r}}$.

Because the signal $s_{r}(\eta)$ is related to the Fourier transform of impulses located at point reflector positions, the above problem becomes analogous to the problem of recovering a 
signal from the magnitude of its Fourier transform which is a relatively well studied area [8], [9], [10]. Unfortunately there exists no closed form solution for this problem. Also, majority of work are on the solution of the problem for real valued images, which is not well suited to the SAR area in which the image data is complex valued.

Here, we use the PSO technique to find $D_{k}^{\prime} s$ and $\omega_{k}^{\prime} s$ of complex exponentials whose sum has the same magnitude as $s_{r}(\eta)$. However, note that the solution to this problem is not unique because of the well-known Fourier transform relations given below:

$$
\begin{gathered}
g(t) \stackrel{\mathcal{F}}{\longrightarrow} \mathcal{G}(\omega), \\
g^{*}(-t) \stackrel{\mathcal{F}}{\longrightarrow} \mathcal{G}(\omega)^{*}, \\
|\mathcal{G}(\omega)|=\left|\mathcal{G}(\omega)^{*}\right| .
\end{gathered}
$$

From the above relation, we can conclude that if a signal is a solution, then its time-inverted and conjugated version is also a solution. In addition, if there exists a solution then its time shifted versions are also in the solution set.

In order to restrict the solution set, any one of the complex exponentials can be fixed at zero frequency by taking it to a common parenthesis:

$$
\begin{gathered}
\left|\tilde{s}_{r}(\eta)\right|=\left|D_{1} e^{j \omega_{1} \eta}+\cdots+D_{N} e^{j \omega_{N} \eta}\right| \\
=\left|e^{j \omega_{1} \eta} \hat{s}_{1}(\eta)\right|=\left|e^{j \omega_{2} \eta} \hat{s}_{2}(\eta)\right|=\cdots=\left|e^{j \omega_{N} \eta} \hat{s}_{N}(\eta)\right|,
\end{gathered}
$$

where,

$$
\begin{gathered}
\hat{s}_{1}(\eta)=\left(D_{1}+D_{2} e^{j\left(\omega_{2}-\omega_{1}\right) \eta}+\cdots+D_{N} e^{j\left(\omega_{N}-\omega_{1}\right) \eta}\right), \\
\hat{s}_{2}(\eta)=\left(D_{1} e^{j\left(\omega_{1}-\omega_{2}\right) \eta}+D_{2}+\cdots+D_{N} e^{j\left(\omega_{N}-\omega_{2}\right) \eta}\right), \\
\vdots \\
\hat{s}_{N}(\eta)=\left(D_{1} e^{j\left(\omega_{1}-\omega_{N}\right) \eta}+D_{2} e^{j\left(\omega_{2}-\omega_{N}\right) \eta}+\cdots+D_{N}\right) .
\end{gathered}
$$

Both $\hat{s}_{1}(\eta), \hat{s}_{2}(\eta), \ldots, \hat{s}_{N}(\eta)$ and their corresponding inverted/conjugated (showed as $\hat{s}_{1}^{*}(-\eta), \hat{s}_{2}^{*}(-\eta), \ldots, \hat{s}_{N}^{*}(-\eta)$ ) counterparts are solutions to our magnitude equivalance problem:

$$
\begin{gathered}
\left|s_{r}(\eta)\right|=\left|\hat{s}_{1}(\eta)\right|=\cdots=\left|\hat{s}_{N}(\eta)\right| \\
\left|\hat{s}_{1}^{*}(-\eta)\right|=\cdots=\left|\hat{s}_{N}^{*}(-\eta)\right| .
\end{gathered}
$$

Thus, we have total number of $2 N$ signals (having one exponential component set to zero frequency) satisfying the above amplitude equivalency condition. By restricting the original problem to this set with $2 N$ possible solutions, we clearly lost the absolute frequency information corresponding to absolute azimuth positions of point reflectors. However frequency differences corresponding to relative azimuth positions of point reflectors with respect to each other remains the same among the set of equivalent solutions. One possible solution to get the absolute positions of targets would be to subtract the linear phase term from the estimated phase error. This approach runs well with the assumption that the phase error does not have a linear trend which is generally the case.
Applying PSO algorithm to find $\hat{s}(\eta)$, any one of $2 N$ signals may result as a solution. To get the phase error estimates corresponding to these solutions, we subtract the phase of the estimated signal from the phase of original signal as shown below:

$$
\begin{gathered}
\hat{\phi}_{e i}(\eta)=\angle s_{r}(\eta)-\angle \hat{s}_{i}(\eta) \\
\hat{\phi}_{e i}(\eta)=\angle\left(\sum_{i=1}^{N}\left|C_{i}\right| e^{j \omega_{i} \eta+\angle C_{i}}\right)+\phi_{e}(\eta)- \\
\angle\left(\sum_{k=1}^{N}\left|D_{k}\right| e^{j \omega_{k} \eta+\angle D_{k}}\right) .
\end{gathered}
$$

The phase estimate can be written in a more compact form as;

$$
\hat{\phi}_{e i}(\eta)=\phi_{e}(\eta)+G(\eta)
$$

where,

$$
\begin{gathered}
G(\eta)=\angle\left(\sum_{i=1}^{N}\left|C_{i}\right| e^{j \omega_{i} \eta+\angle C_{i}}\right)- \\
\angle\left(\sum_{k=1}^{N}\left|D_{k}\right| e^{j \omega_{k} \eta+\angle D_{k}}\right) .
\end{gathered}
$$

If $G(\eta)$ is zero, a constant or a linear function of $\eta$, then the estimated phase error matches with the actual phase error and the corrected SAR image is freed from blurs caused by uncompensated motion errors. Otherwise estimated phase error differs from the actual one by the function $G(\eta)$. Then, the estimated phase error term as appeared in $s_{r}(\eta)$ is;

$$
e^{j \hat{\phi}_{e i}(\eta)}=e^{j \phi_{e}(\eta)} e^{j G(\eta)} \text {. }
$$

The final corrected image deteriorates with a convolution by the Fourier transform of the above estimated phase error. The Fourier transform of the estimated phase error is the convolution of two terms;

$$
F\left\{e^{j \hat{\phi}_{e i}(\eta)}\right\}=F\left\{e^{j \phi_{e}(\eta)}\right\} * F\left\{e^{j G(\eta)}\right\} .
$$

In the above equation, convolving with the second term spreads the original phase error so the estimated phase error widens in the azimuth direction. Taking advantage of this observation we use entropy, in order to differentiate the correct phase error estimation from other wrong estimates. Because, the estimated phase error is wider if it does not correspond to the correct one, the entropy of the Fourier transform of the estimated phase error corresponding to correct solution always gives a low value comparing to the other possible solutions. Based on this observation we develop a max-toentropy ratio metric to discern the correct solution among other possible solutions. The correct solution (among all other possible solutions) has the greatest value of the max-to-entropy ratio as formulated below:

$$
\hat{\phi}_{e}(\eta)=\max _{\arg \hat{\phi}_{e i}(\eta)}\left\{\frac{\max \left|F\left\{e^{j \hat{\phi}_{e i}(\eta)}\right\}\right|}{H\left|F\left\{e^{j \hat{\phi}_{e i}(\eta)}\right\}\right|}\right\},
$$

where, $H$ represents entropy and $F\{$.$\} represents Fourier$ transform in the above equation. 


\section{ApPlication of PSO Algorithm}

In our method, PSO algorithm is used to find one of the signals, $\hat{s}_{i}(\eta)$, where $|s(\eta)|=\left|\hat{s}_{1}(\eta)\right|=\cdots=\left|\hat{s}_{N}(\eta)\right|$. Let's re-write $\hat{s}_{1}(\eta)$ below for reference,

$$
\hat{s}_{1}(\eta)=\left(D_{1}+D_{2} e^{j\left(\omega_{2}-\omega_{1}\right) \eta}+\cdots+D_{N} e^{j\left(\omega_{N}-\omega_{1}\right) \eta}\right) .
$$

In $\hat{s}_{1}(\eta)$, we have $N$ unknown complex amplitudes, $D_{1}, D_{2}$, $\ldots, D_{N}$, and $N-1$ unknown frequencies, $\left(\omega_{2}-\omega_{1}\right),\left(\omega_{3}-\omega_{1}\right)$, $\ldots,\left(\omega_{N}-\omega_{1}\right)$. Thus, there are total number of $3 N-1$ unknown real parameters for PSO algorithm.

While applying PSO, we generally do not know the actual number $(N)$ of point targets residing in the interested part of the scene. In such cases, we can start to run PSO algorithm with a number, say $M$ such that $M>N$. This approach, although increases computation time, finds the correct solutions for $N$ point targets and assigns zero to magnitudes of the rest of $M-N$ artificial point targets.

The fitness function, we used in the PSO algorithm, is the square of $L_{2}$ norm of the magnitude differences between $s_{r}(\eta)$ and $\hat{s}_{i}(\eta)$,

$$
f=\sum_{\eta}\left(\left|s_{r}(\eta)\right|-\left|\hat{s}_{i}(\eta)\right|\right)^{2} .
$$

By using the above fitness function, we try to minimize the energy of the error between the original and estimated signals. PSO algorithm has many varieties. In our work we adapted the standard PSO algorithm defined by [11]. It includes a local ring topology, non-uniform swarm initializations, and 50 particles. In a great proportion of all trials, PSO algorithm converges to one of $2 N$ solutions. In some cases with very small proportion, algorithm stuck to local minima and do not converge to a feasible solution. To prevent these cases, a limit is set to the total number of loops executed. If the limit is reached before the targeted error is obtained, then the algorithm starts a new trial. By using the converged solution, all solution set can be constructed by

$$
\begin{gathered}
\hat{s}_{2}(\eta)=\frac{\hat{s}_{1}(\eta)}{e^{j\left(\omega_{2}-\omega_{1}\right) \eta}} \\
\vdots \\
\hat{s}_{N}(\eta)=\frac{\hat{s}_{1}(\eta)}{e^{j\left(\omega_{N}-\omega_{1}\right) \eta}} .
\end{gathered}
$$

To get phase error estimates corresponding to these solutions, we subtract the phase of the estimated signal from the phase of the original signal as shown below,

$$
\begin{gathered}
\hat{\phi}_{e 1}(\eta)=\angle s(\eta)-\angle \hat{s}_{1}(\eta) \\
\vdots \\
\hat{\phi}_{e N}(\eta)=\angle s(\eta)-\angle \hat{s}_{N}(\eta) \\
\hat{\phi}_{e(N+1)}(\eta)=\angle s(\eta)-\angle \hat{s}_{1}^{*}(-\eta) \\
\vdots \\
\hat{\phi}_{e 2 N}(\eta)=\angle s(\eta)-\angle \hat{s}_{N}^{*}(-\eta)
\end{gathered}
$$

From this solution set we choose the one with the greatest max-to-entropy ratio as the correct solution.

As an example we applied the PSO algorithm to the following scenario. There are three point scatterers at the same range bin located next to each other in azimuth direction. The raw data with phase error is compressed in range and in azimuth to obtain the SAR image. The range cut of the image through azimuth direction is given in Figure 1. As seen from the figure, it is highly distorted in the azimuth direction. PSO algorithm is

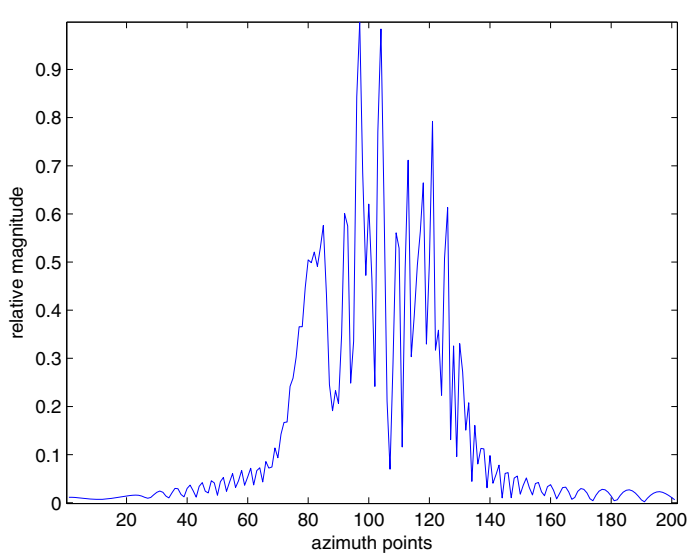

Fig. 1. Range cut of an image with phase error.

applied to estimate the phase error. Then the estimated phase error is corrected and the range cut of reconstructed image is given in Figure 2. The image is correctly focused and three point reflectors are clearly discernible.

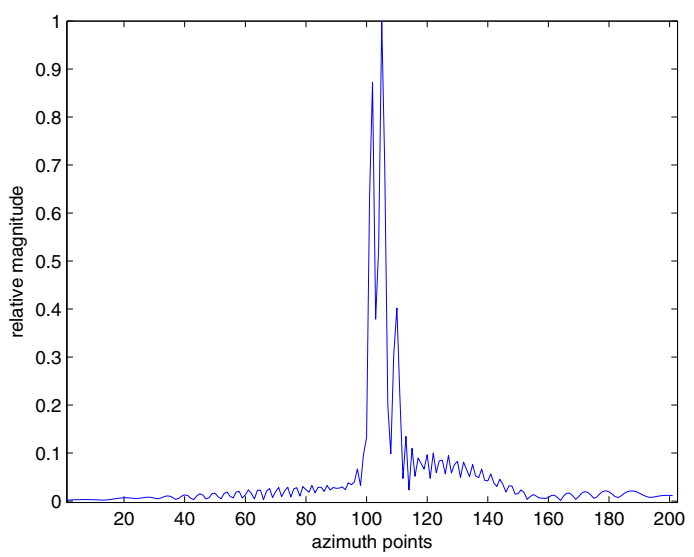

Fig. 2. Range cut of corrected image.

For comparison, the actual and estimated phase errors are displayed in Figure 3. As seen from the figure the PSO algorithm is applied successfully to estimate phase errors of the SAR raw data. The result of another trial of the PSO technique to the same data is given in Figure 4. This solution corresponds to the time inverted/conjugated signal as described in section 3. The max-to-entropy ratios corresponding to 


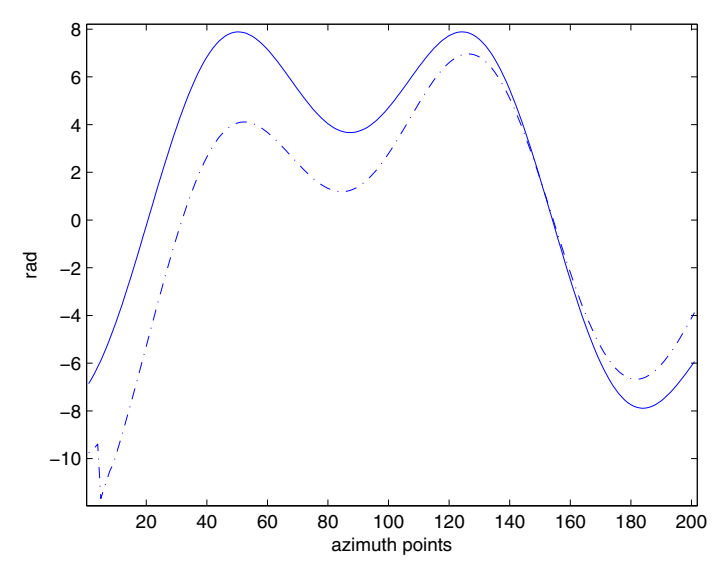

Fig. 3. Actual (-) and estimated (-.-) phase errors.

signals of Figures 2 and 4 are 26.68 and 20.94 respectively which clearly indicates that the Figure 2 corresponds to the correct solution.

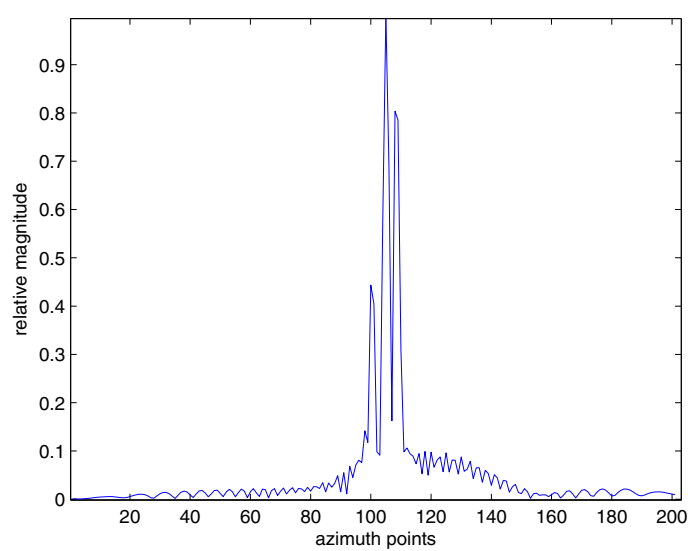

Fig. 4. Range cut of mirror image.

\section{CONCLUSION}

In this study, we applied PSO algorithm to the problem of SAR phase error estimation. We model targets as closely spaced point reflectors and exploit just the magnitude of the received signal. In this respect, we set an analogy between our problem and the problem of estimating signals whose only the Fourier transform magnitude is known. Application of PSO to this problem gives reasonable results. To be used in SAR data corresponding to real scenes, the technique needs to be applied to different range bins containing different targets. But data for widely spaced scatterers residing in the same range bin should be discarded from the problem set because they violate the assumption that the phase errors are the same for targets residing in the same range bin. Then the phase errors are estimated for the remaining targets and these estimates are used to compensate for the neighboring range bins.

\section{ACKNOWLEDGEMENTS}

This research is funded in part by Meteksan Savunma Sanayii A.Ş. under contract no. 106A030.

\section{REFERENCES}

[1] P. H. Eichel, D. C. Ghiglia and C. V. Jakowatz, "Speckle processing method for synthetic aperture radar phase correction", Optics Letters, vol. 14, pages 1-3, October 1989.

[2] D. E. Wahl, P. H. Eichel, D. C. Ghiglia and C. V. Jakowatz, "Phase gradient autofocus - A robust tool for high resolution SAR phase correction", IEEE Transactions on Aerospace and Electronic Systems, vol. 30, pages 827-835, October 1994.

[3] J. Kennedy and R. Eberhart, "Particle Swarm Optimization", IEEE International Conference on Neural Networks, pages 1942-1948, 1995.

[4] J. T. Parker and J. Norgard, "Autofocusing for RF Tomography Using Particle Swarm Optimization”, IEEE Radar Conference, 2008. RADAR'08, pages 1-6, 2008.

[5] T. S. Lim, V. C. Koo, H. T. Ewe and H. T. Chuah, "High-frequency phase error reduction in SAR using particle swarm optimization algorithm", J. of Electromagn. Waves and Appl., vol.21, pages 795-810, 2007.

[6] T. S. Lim, V. C. Koo, H. T. Ewe and H. T. Chuah, "A SAR autofocus algorithm based on particle swarm optimization", Progress In Electromagnetics Research B, vol. 1, pages 159-176, 2008.

[7] I. G. Cumming and F. H. Wong, Digital Processing Of Synthetic Aperture Radar Data: Algorithms And Implementation, Artech House Inc., pages 231-232, 2005.

[8] R. G. Lane, W. R. Fright and R. H. T. Bates, "Direct Phase Retrieval", IEEE Transactions on Acoustics, Speech, and Signal Processing, vol.ASSP-35, pages 520-526, April 1987.

[9] A. V. Oppenheim, J. S. Lim and S. R. Curtis, "Signal Synthesis and Reconstruction from Partial Fourier-domain Information", J. Opt. Soc. Am., vol.73, pages 1413-1420, November 1983.

[10] M. H. Hayes, J. S. Lim and A. V. Oppenheim, "Signal Reconstruction from Phase or Magnitude", IEEE Transactions on Acoustics, Speech, and Signal Processing, vol. ASSP-28, pages 672-680, April 1980.

[11] D. Bratton and J. Kennedy, "Defining a Standard for Particle Swarm Optimization", Proceedings of the 2007 IEEE Swarm Intelligence Symposium, pages 120-127, 2007. 